\title{
Insights into Structural Evolution of a Solid Electrolyte Interphase Using Thin Window Si Membrane Negative Electrodes
}

\author{
V.P. Oleshko ${ }^{1}$, S. Takeuchi ${ }^{2,5}$, W.R. McGehee ${ }^{3}$, E. Strelcov ${ }^{3,4}$, D. Gundlach, ${ }^{2}$ N. Zhitenev ${ }^{3}$, C.L. Soles ${ }^{1}$, \\ J.J. McClelland ${ }^{3}$ \\ ${ }^{1}$ Material Measurement Laboratory, ${ }^{2}$ Physical Measurement Laboratory, and ${ }^{3}$ Center for Nanoscale \\ Science and Technology, National Institute of Standards and Technology, Gaithersburg, MD 20899 \\ ${ }^{4}$ Institute for Research in Electronics \& Applied Physics, University of Maryland, College Park, MD \\ 20742
}

${ }^{5}$ Theiss Research, La Jolla, CA 92037

Silicon has emerged as one of the most promising anode materials for high-performance Li-ion batteries because of its exceptional theoretical specific capacity of $4200 \mathrm{mAh} \mathrm{g}^{-1}$, which is more than 10 times the theoretical capacity of graphite $(372 \mathrm{mAh} / \mathrm{g})[1,2]$. However, practical use of Si as a negative electrode is hampered by high volume expansion of up to $400 \%$ on full lithium insertion, pulverization of the material, and growth of a solid electrolyte interphase (SEI) [1,2]. Physico-chemical stability of the SEI at the interface between $\mathrm{Si}$ and the liquid electrolyte is a crucial factor in achieving a long cycle life. In this work, we focus on phase structural evolution of the SEI during lithiation of electron transparent $c$-Si anodes, which allow correlation of electrochemical properties with the structure on the same electrode feature. We have chosen for this study $p$-doped thin window $<100>$-oriented single crystalline planar $c$ Si membranes as model electrodes suitable for monitoring lithiation processes and characterization by high-resolution analytical S/TEM. A $\mathrm{LiClO}_{4}$ solution in a 1:1 mixture by volume of ethylene carbonate (EC) : diethyl carbonate (DEC) was employed as a nonaqeuous electrolyte due to its low viscosity and anodic stability.

Electrochemical measurements were carried out in a three-electrode custom-designed PTFE cell. The working electrode was a $3 \mathrm{~mm}$ diameter $c$-Si membrane that had nine $35 \mathrm{~nm}$-thick windows. The membranes were connected to the current collector by a $\mathrm{Cu}$ lead. The counter and reference electrodes were made from a lithium foil. Cyclic voltammetry of the Si membranes in a $1 \mathrm{M}$ electrolyte was carried out from open circuit voltage and between $0.01 \mathrm{~V}$ and $2.0 \mathrm{~V}$ vs. $\mathrm{Li} / \mathrm{Li}^{+}$with the sweep rate of $0.1 \mathrm{mV} \mathrm{sec}$ ${ }^{1}$. Lithiated membranes were held $17 \mathrm{~h}$ at $0.15 \mathrm{~V}$. After electrochemical lithiation, the samples were transferred for examination in a TEM. A glove bag purged with an UHP Ar was used to prevent the samples from being exposed to an ambient environment during the transfer. Low-dose BF- and DF- TEM, STEM, HRTEM, SAED, EELS and EDXS were applied ex situ to characterize the morphology, crystallinity and local chemical compositions of the passivation films formed during lithiation/delithiation of the membrane anodes. Soaking of a control Si membrane into the electrolyte for 24 hours without processing did not reveal the growth of the SEI.

The positions of the reduction peaks at $1.5 \mathrm{~V}, 1.3 \mathrm{~V}$ and $0.6 \mathrm{~V}$ in a cyclic voltammogram (Fig. 1) were in good agreement with the positions of the reduction peaks obtained for a $1 \mathrm{M} \mathrm{LiClO}_{4} / \mathrm{EC}$ : dimethyl carbonate (DMC, 1:1 by vol.) with a Pt electrode [2] and can be attributed to decomposition of the electrolyte. Our findings suggest that the SEI consists of an inner "inorganic" layer composed of $\mathrm{Li}_{2} \mathrm{O}$, $\mathrm{Li}_{2} \mathrm{CO}_{3}, \mathrm{LiOH}$, as well as $\mathrm{Li}_{\mathrm{x}} \mathrm{SiO}_{\mathrm{y}}$ formed from a native oxide and an outer preferentially "organic" layer comprising mostly alkyl carbonates (Fig. 1b) [2,3]. Si lithiation with the formation of a $\mathrm{Li}_{1.7} \mathrm{Si}$ phase occurs at $0.33 \mathrm{~V} \mathrm{[4]} \mathrm{and} \mathrm{an} \mathrm{increase} \mathrm{of} \mathrm{the} \mathrm{current} \mathrm{intensity} \mathrm{below} 0.3 \mathrm{~V}$ can be attributed to lithiation of the $\mathrm{Si}$ matrix. The oxidation peaks at $0.7 \mathrm{~V}$ and $1.5 \mathrm{~V}$ can be attributed to delithiation of $\mathrm{Si}$ and $\mathrm{SiO}_{\mathrm{x}}$, respectively [5]. For the $2^{\text {nd }}$ cycle, the reduction peak at $1.5 \mathrm{~V}$ is not as prominent as for the 1 st cycle. However, reduction current is still detected suggesting that the SEI continues to grow during the $2^{\text {nd }}$ cycle. 
Observed SEI features include various morphological shapes, such as dendritic sponge aggregates impregnated with a liquid electrolyte (Fig. 2a). By soaking a lithiated Si membrane in DMC, web-like organic-inorganic structures grown on the $c$-Si surface and agglomerates of $\mathrm{LiClO}_{4}$ nanocrystallites were found (Fig. 2b). Ex situ BF-TEM (Figs. 2a-b) and HRTEM (Fig. 2c) studies indicate that dynamically evolving SEI growth profoundly dominates over $c$-Si lithiation. An island-like growth of such loose nonuniform SEI films can cause a rise of the interfacial impedance and low Coulombic efficiency, thus essentially hampering the potential of $\mathrm{Si}$ anodes to be practically realized.

References: [1] V.P. Oleshko, et al., Microsc. Microanal. 22(Suppl.3), (2016) 1556. [2] R. Marom, et al., J. Electrochem. Soc., 157(2010) A972. [3] M. Hess, Electrochimica Acta, 244 (2017) 69. [4] J. Wen, R. A. Huggins, J. Solid State Chem., 37(1981) 271. [5] Q. Sun, et al., Appl. Surf. Science, 254 (2008) 3774

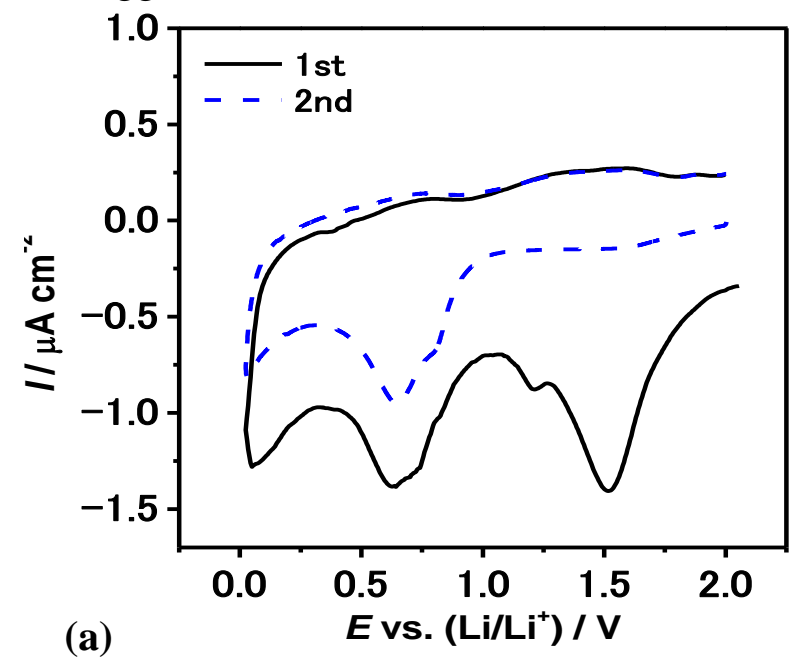

(b)

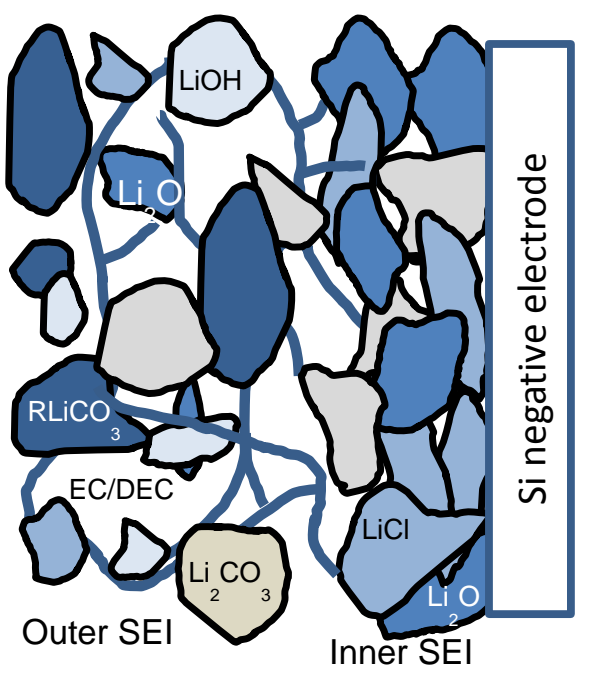

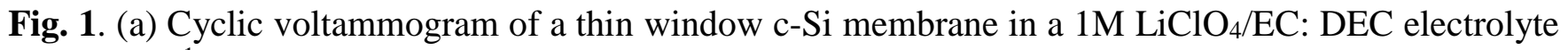
at $0.1 \mathrm{mVs}^{-1}$ scan rate. (b) Schematic model of an SEI comprising a 10 to $100 \mathrm{~nm}$ thick outer organicinorganic polymeric layer and a $2-10 \mathrm{~nm}$-thick inner inorganic layer, respectively.

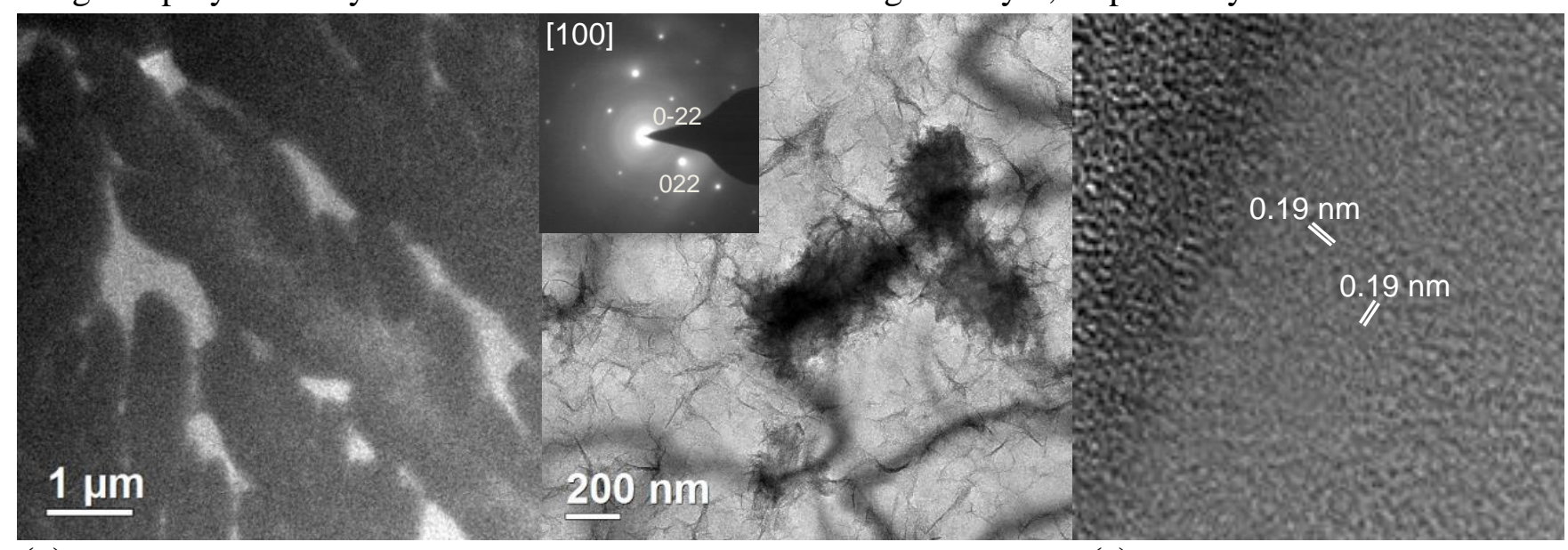

(a)

(b)

(c)

Fig. 2. Ex situ low-dose BF-TEM. (a) Dendritic sponge aggregates impregnated with a liquid electrolyte in an outer SEI layer after $17 \mathrm{~h}$ at $1.5 \mathrm{~V}$ cutoff. (b) Web-like aggregated structures formed after drying the electrolyte. SAED pattern (upper inset) of a lithiated $c$-Si membrane at [100] zone axis. (c) HRTEM, a densified inner SEI layer of the lithiated membrane, (002) Si lattice fringes are barely visible through the amorphous SEI film. 